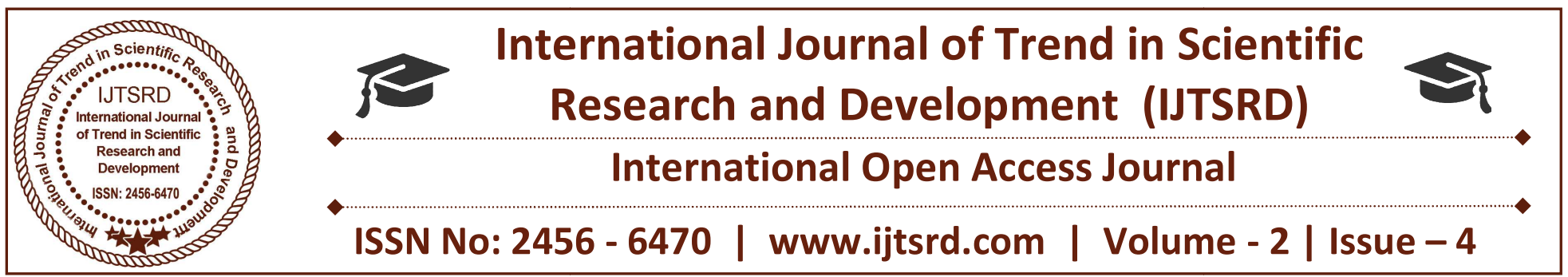

\title{
An Advanced New Adaptive Alpha-Trimmed Median Filter for Removel of Salt and Pepper Noises for Recovering the Image Protecting Edge Data
}

\author{
Sathuluri Ramarao \\ M.Tech Scolar, Digital Image Processing, Nalanda \\ Institute of Engineering and Technology (NIET), \\ Guntur District, Andhra Pradesh, India
}

\begin{abstract}
L. Srinivas
Assistant Professor, Digital Image Processing, Nalanda Institute of Engineering and Technology

(NIET), Guntur District, Andhra Pradesh, India
\end{abstract}

\section{ABSTRACT}

Here in this paper we discuss about an advanced new adaptive alpha-trimmed median filter for removel of salt and pepper noises for recovering the image protecting edge data. In olden days we used anlage technology to capture the images which manes photo reals or negatives to capture the images, even though they have high resolution when compared when compared to the today digital images, we have to compromise the parameters like lighting, and some other noises. When compared to the disadvantages of the analogue technology digital technology is preferred. In the digital technology we can edit or modify or even we can compress the images without losing visual effects. When coming to the digital technology there are some complications like noise, size issue, etc. While considering the noise there are different types of noises like Salt and Pepper noise, Gaussian noise, etc. Salt and Pepper noise is the frequently observed noise, in which some the pixel values are disturbed and changes into pure white or pure black and while considering the colour images it changes maximum or minimum of corresponding colour. This creates the disturbances in the images in different levels. Higher the damaged pixels higher the noise appears. In our proposed system we are using a new filter called Alpha trimmed median filter. The pixels which effected by noise have the value 0 or 255 which are black and white respectively. In olden methods they mostly used the median filter to rectify or recover the noise images, even though they give the resultant output in rectifications it also results in the loss of edge data. In our proposed system we are changing the value of only corrupted pixel values which results in recovering the image and protecting the edge data.

\section{INTRODUCTION}

Photographs are corrupted via noise mainly all through cimage acquisition and/or transmission. Impulse noise is one of these noises that is generated all through imaging because of defective switching wherein brief transients are gift. Impulse noise denoising is a crucial problem in case of digital photo processing. The arrival of image may be considerably affected even at low density of impulse noise.

There are varieties of impulse noise, they may be salt and pepper noise and random valued noise. In case of salt and pepper noise the corrupted pixel takes the price of maximum or minimal gray stage. Removal of noises in snap shots required earlier than going for exclusive form of processing including segmentation, edge detection and item popularity. There are numerous nonlinear methods for elimination of salt and pepper noise. In previous few a long time median filters (MF) attracted many due to its part keeping and simplicity nature. The downside of using standard median filter is it really works for most effective low densities of noise. Some other disadvantage of traditional median filter, it plays uniformly during the photograph regardless of whether or not the image 
pixel is corrupted or not. When noise density will become high the brink info are not preserved.

Adaptive median filter out (AMF) affords desirable outcomes for low densities of noise however for excessive density of noise window length is to be accelerated. In switching median clear out, the selection is based on a pre-defined threshold value. The essential downside of this technique is that the performance of this method lies on a terrific decision which is very difficult. The boom within the length of window ends in blurring of the photograph. Choice based totally set of rules (DBA) offers better outcomes than the median and adaptive median filters. In this case first the processing pixel is checked whether it's far corrupted or uncorrupted, if it is uncorrupted then its miles left unchanged otherwise its miles changed by the median of the $3 \times 3$ window. If the median is again noisy i.e. has a value of 0 or 255 then it is replaced.

With the previously processed pixel price. However repeated substitute results in streaking impact. In this paper, we've got proposed a brand new filtering approach that can take away salt and pepper noise extra successfully from the photograph. This filter out works nicely even in the case while the input image could be very enormously corrupted and additionally preserves the image information. This paper is prepared as follows. Segment 11 affords the proposed technique.

When compared to the disadvantages of the analogue technology digital technology is preferred. In the digital technology we can edit or modify or even we can compress the images without losing visual effects. When coming to the digital technology there are some complications like noise, size issue, etc. While considering the noise there are different types of noises like Salt and Pepper noise, Gaussian noise, etc. Salt and Pepper noise is the frequently observed noise, in which some the pixel values are disturbed and changes into pure white or pure black and while considering the colour images it changes maximum or minimum of corresponding colour. This creates the disturbances in the images in different levels. Higher the damaged pixels higher the noise appears. In our proposed system we are using a new filter called Alpha trimmed median filter. The pixels which effected by noise have the value 0 or 255 which are black and white respectively. In olden methods they mostly used the median filter to rectify or recover the noise images, even though they give the resultant output in rectifications it also results in the loss of edge data. In median filter process the corresponding pixel value is changed with the average or mean value of the surrounding pixels, which results in loss of edge data. In our proposed system we are changing the value of only corrupted pixel values which results in recovering the image and protecting the edge data.

\section{IMAGE PROCESSING}

Digital Image processing, the manipulation of snap shots via pc, is pretty recent improvement in phrases of man's ancient fascination with visible stimuli. In its short records, it has been carried out to nearly each sort of photos with varying diploma of success. The inherent subjective enchantment of pictorial presentations draws perhaps a disproportionate quantity of attention from the scientists and also from the layman. Virtual image processing like different glamour fields, suffers from myths, mis-connect ions, mis-understandings and mis-records. Its far huge umbrella underneath which fall various thing of optics, electronics, mathematics, pictures pics and laptop technology. Its miles really multidisciplinary undertaking ploughed with imprecise jargon.

Several component integrate to suggest a lively destiny for digital photo processing. A chief aspect is the declining cost of pc gadget. Several new technological tendencies promise to similarly sell digital photograph processing. These include parallel processing mode practical via low cost microprocessors, and the usage of charge coupled gadgets (CCDs) for digitizing, storage at some point of processing and display and massive low fee of photo storage arrays.

\section{A. Image acquisition}

Image Acquisition is to acquire a digital image. To do so requires a picture sensor and the capability to digitize the sign produced by means of the sensor. The sensor might be monochrome or shade television digital camera that produces a whole photo of the hassle area every $1 / 30 \mathrm{sec}$. the image sensor may also be line scan camera that produces a single photo line at a time. In this case, the objects motion past the line. 


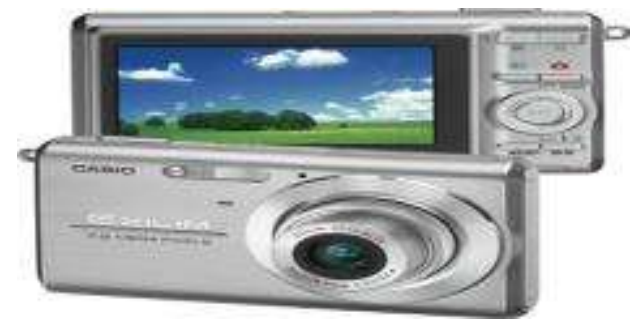

Figure 1: Digital Camera

Scanner produces a -dimensional photograph. If the output of the digital camera or other imaging sensor is not in virtual form, an analogue to digital converter digitizes it. The character of the sensor and the photograph it produces are decided via the software

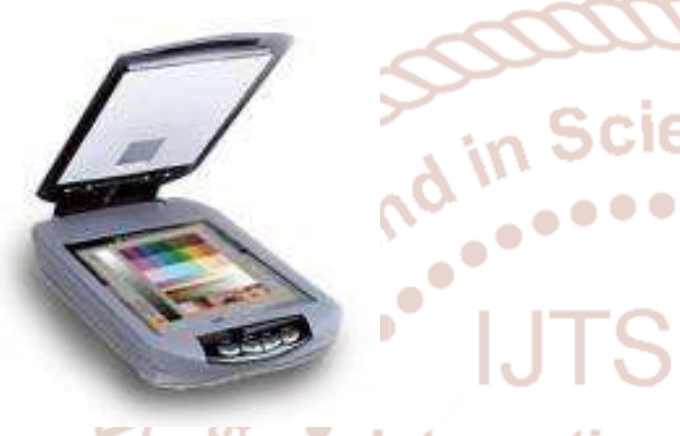

Figure 2: Image Scanner

\section{B. Image enhancement}

Picture enhancement is many of the most effective and most attractive areas of digital photograph processing. Basically, the concept at the back of enhancement techniques is to convey out element that is obscured, or truly to spotlight certain functions of thrilling a photo. A familiar instance of enhancement is whilst we increase the comparison of a photo due to the fact "it appears better." it is important to remember the fact that enhancement is a totally subjective region of picture processing.

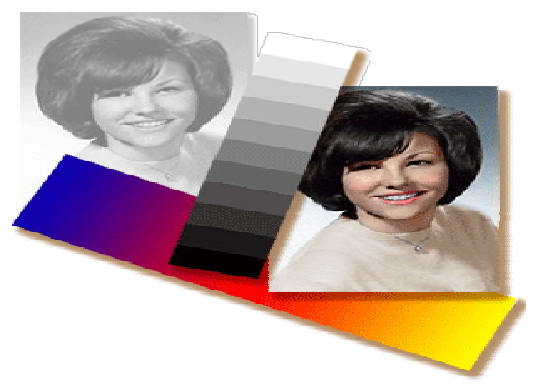

Figure 3: Enhanced Image

\section{Image restoration}

Picture healing is a place that still offers with improving the appearance of photograph but, not like enhancement, that's subjective, image recovery is objective, in the feel that restoration strategies tend to be based totally on mathematical or probabilistic fashions of photograph degradation.

Enhancement, on the other hand, is based on human subjective possibilities concerning what constitutes a "true" enhancement result. For example, assessment stretching is taken into consideration an enhancement method because it is based usually on the beautiful aspects it might gift to the viewer, wherein as removal of picture blur by applying a de-blurring function is considered a healing method.

\section{Colour image processing}

The usage of colour in picture processing is motivated by means of fundamental elements. First, color is an effective descriptor that often simplifies item identification and extraction from a scene. 2nd, humans can determine heaps of coloration sun shades and intensities, compared to approximately most effective dozen sunglasses of grey. This $2 d$ thing is especially critical in guide photo analysis.

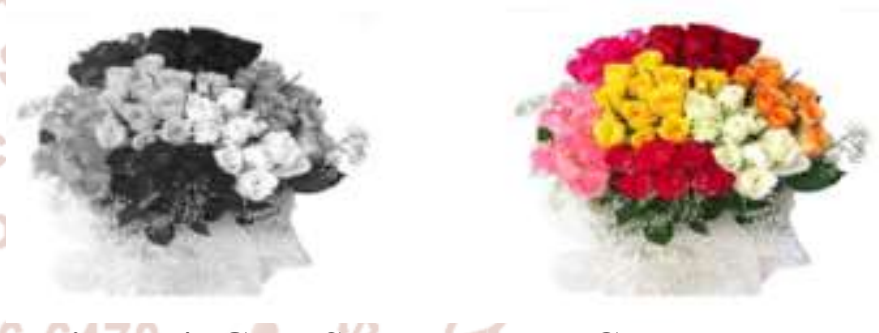

Figure 4: Gray Scale Image to Colour Image

\section{E. Wavelets and multi resolution processing}

Wavelets are the formation for representing pix in various ranges of resolution. although the Fourier remodel has been the mainstay of remodel primarily based photograph processing because the late1950's, an extra latest transformation, referred to as the wavelet rework, and is now making it even easier to compress, transmit, and examine many images. In contrast to the Fourier transform, whose foundation features are sinusoids, wavelet transforms are primarily based on small values, called Wavelets, of varying frequency and limited length. 


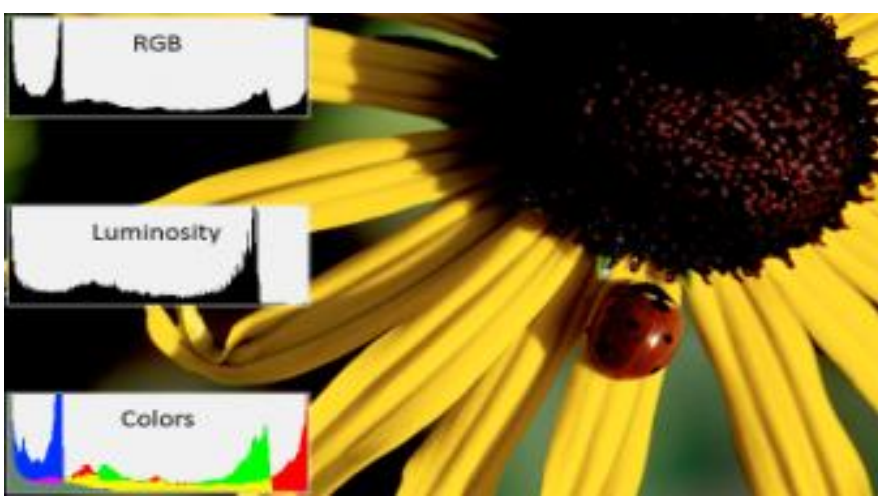

Figure 5: Wavelets and multi resolution Image

Wavelets were first proven to be the foundation of a powerful new method to signal processing and evaluation known as Multi decision concept. Multi decision theory carries and unifies strategies from an expansion of disciplines, including sub band coding from sign processing, quadrature reflect filtering from virtual speech popularity, and pyramidal photo processing.

\section{F. Compression}

Compression, because the name implies, deals with strategies for decreasing the garage required saving an image, or the bandwidth required for transmitting it. Although garage era has stepped forward extensively over the past decade, the same can't be said for transmission ability. That is actual mainly in makes use of the net, which might be characterized through widespread pictorial content. Image compression is acquainted to maximum customers of computer systems within the form of photograph file extensions, along with the jpg record extension used within the JPEG (Joint Photographic Experts group) picture compression widespread.

\section{G. Morphological processing}

Morphological processing offers with tools for extracting photograph additives that are beneficial within the illustration and outline of shape. The language of mathematical morphology is about concept. As such, morphology gives a unified and powerful method to numerous image processing issues. Units in mathematical morphology represent gadgets in picture. For example, the set of all black pixels in a binary image is a complete morphological description of the picture.

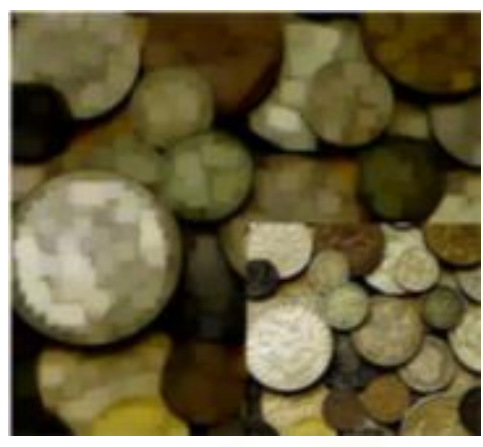

\section{Figure 6: Morphological Image}

In binary photographs, the sets in question are individuals of the 2-D integer area $\mathrm{Z2}$, where every detail of a hard and fast is a 2-D vector whose coordinates are the $(\mathrm{x}, \mathrm{y})$ coordinates of a black(or white) pixel inside the photo.

Gray-scale digital photos can be represented as units whose components are in Z3. In this example, two components of every detail of the set talk over with the coordinates of a pixel, and the 0.33 corresponds to its discrete grey-degree cost.

\section{H. Segmentation}
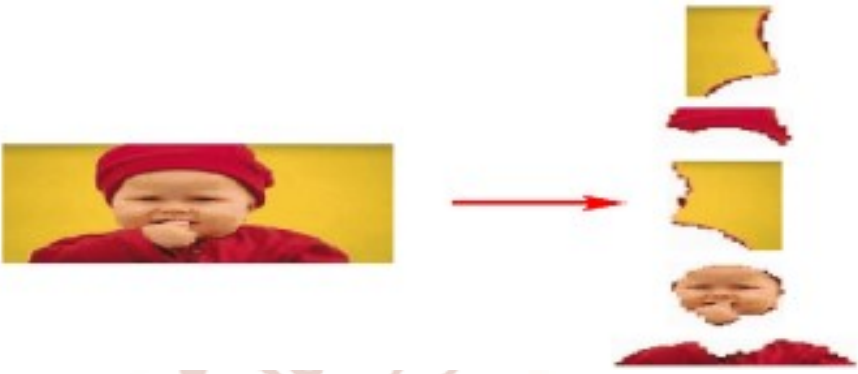

\section{Figure 7: Image Segmentation}

However, vulnerable or erratic segmentation algorithms nearly continually assure eventual failure. In fashionable, the extra correct the segmentation, the more likely popularity is to be triumphant.

\section{REPRESENTATION DESCRIPTION}

AND

Illustration and description almost always follow the output of a segmentation stage, which generally is uncooked pixel records, constituting either the boundary of a place (i.e., the set of pixels setting apart one photo location from another) or all the factors inside the place itself.

The first selection that must be made is whether or not the records must be represented as a boundary or as a complete area. Boundary illustration is appropriate 
when the focal point is on external shape traits, consisting of corners and inflections.

Regional representation is suitable when the focus is on internal properties, together with texture or skeletal shape. In some programs, these representations complement each different. Selecting a representation is only part of the solution for reworking uncooked information into a shape appropriate for next computer processing. A technique have to additionally be special for describing the statistics in order that functions of interest are highlighted. Description, additionally known as feature selection, deals with extracting attributes that bring about some quantitative facts of interest or are primary for differentiating one magnificence of objects from any other.

\section{A. Object recognition}

The last level involves recognition and interpretation.

\section{B. Knowledgebase}

Information about a problem area is coded into photograph processing device inside the shape of an information database. This understanding may be as simple as detailing areas of an image while the records of pursuits is understood to be positioned, thus proscribing the quest that must be carried out in in search of that information. The expertise base additionally can be quite complex, including an inter related to listing of all fundamental viable defects in a substances inspection problem or an image facts base containing excessive resolution satellite $\mathrm{TV}$ for $\mathrm{pc}$ pics of a vicinity in reference to exchange deletion application.

In addition to guiding the operation of every processing module, the expertise base additionally controls the interaction among modules. The device ought to be endowed with the knowledge to apprehend the importance of the place of the string with admire to different components of an address subject. This know-how glides no longer handiest the operation of each module, however it additionally aids in remarks operations among modules through the understanding base. We implemented processing strategies the usage of MATLAB.

\section{Components of an image processing system}

As recently as the mid-Nineteen Eighties, numerous fashions of image processing structures are being sold at some stage in the world had been rather massive peripheral devices that attached to similarly giant host computer systems. Late within the 1980s and early inside the Nineteen Nineties, the marketplace shifted to image processing hardware within the form of unmarried boards designed to be like minded with industry well known buses and to fit into engineering computing device shelves and private computers. Similarly to reducing costs, this marketplace shift also served as a catalyst for an extensive range of new organizations whose strong point is the improvement of software written in particular for photo processing.

\section{Median filter}

In sign processing, it is frequently perfect with a view to carry out a few sort of noise discount on a photograph or signal. The median clear out is a nonlinear digital filtering method, often used to cast off noise. Such noise reduction is a regular preprocessing step to enhance the effects of later processing (for instance, aspect detection on picture). Median filtering is very widely used in virtual photo processing because, under certain conditions, it preserves edges while casting off noise (however see discussion under).

\section{A. Algorithm description}

The main idea of the median clear out is to run through the signal access by using access, changing every access with the median of neighbouring entries. The sample of associates is known as the "window", which slides, access through entry, over the entire sign. For 1D indicators, the maximum obvious window is simply the primary few previous and following entries, whereas for second (or betterdimensional) indicators which include photos, more complex window patterns are possible (which includes "box" or "move" styles). Note that if the window has an odd range of entries, then the median is straight forward to define it's miles just the centre cost after all of the entries inside the window are taken care of numerically. For a good number of entries, there may be a couple of possible median, see median for greater info.

\section{B. Algorithm implementation issues}

Generally, with the aid of some distance the majority of the computational time and effort is spent on calculating the median of each window. Due to the fact the filter out must system every access inside the sign, for big alerts along with pix, the performance of 
this median calculation is an important aspect in figuring out how speedy the algorithm can run.

The "vanilla" implementation defined above types every access inside the window to discover the median; however, seeing that most effective the centre value in a list of numbers is needed, choice algorithms can be tons greater green. Furthermore, some types of signals (very frequently the case for images) use entire quantity representations: in these cases, histogram medians may be a long way more efficient because it is simple to replace the histogram from window to window, and finding the median of a histogram isn't especially onerous.

\section{Edge preservation properties}

Median filtering is one sort of smoothing method, as is linear Gaussian filtering. All smoothing strategies are effective at doing away with noise in smooth patches or smooth areas of a signal, however adversely have an effect on edges. Often although, on the equal time as decreasing the noise in a signal, it's miles important to keep the rims. Edges are of essential significance to the visible look of photos, as an example.

For small to moderate tiers of (Gaussian) noise, the median filter out is demonstrably better than Gaussian blur at getting rid of noise while keeping edges for a given, constant window length. But, its performance is not that a good deal higher than Gaussian blur for excessive tiers of noise, while, for speckle noise and salt and pepper noise (impulsive noise), it's far in particular effective. Due to this, median filtering may be very widely used in virtual photograph processing.

\section{ADAPTIVE FILTER}

An adaptive filter out is a filter out that self-adjusts its transfer feature in line with an optimization algorithm driven with the aid of an errors sign. Through way of assessment, a non-adaptive filter out has a static switch feature. Adaptive filters are required for some packages due to the fact a few parameters of the preferred processing operation (for instance, the locations of reflective surfaces in a reverberate area) are not recognized in advance.

The adaptive clear out makes use of remarks inside the form of an error sign to refine its transfer function to match the changing parameters. Because the strength of digital signal processors has improved, adaptive filters have grown to be plenty greater not unusual and are now robotic ally utilized in devices inclusive of cellular phones and other verbal exchange devices, camcorders and virtual cameras, and scientific tracking equipment.

\section{A. Example application}

Think a medical institution is recording a coronary heart beat (an ECG), which is being corrupted by a 60 $\mathrm{Hz}$ noise (the frequency coming from the power deliver in many countries), but, due to moderate versions within the power deliver to the hospital, the noise sign may include harmonics of the noise and the exact frequency of the noise may range.

The block diagram, shown within the following figure, serves as a basis for precise adaptive filter out realizations, such as Least suggest Squares (LMS) and Recursive Least Squares (RLS). The concept behind the block diagram is that a variable filter out extracts an estimate of the preferred sign.

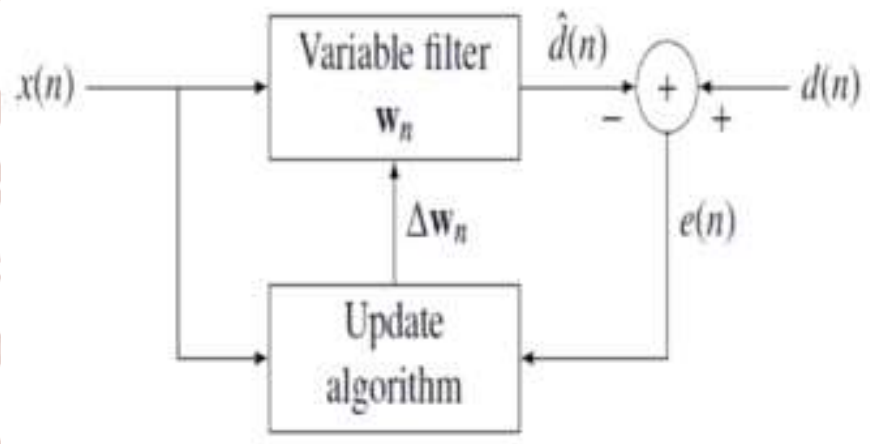

\section{Figure 8: Block Diagram of Adaptive Filter}

To start discussion of block diagram we are taking the following assumptions:

- The input signal is the sum of a desired signal $d(n)$ and interfering noise $v(n)$

$$
x(n)=d(n)+v(n)
$$

- The variable clear out has a Finite Impulse reaction (FIR) structure. For such structures the impulse response is identical to the filter out coefficients. The coefficients for a clear out of order $p$ are described as

$$
\mathbf{w}_{n}=\left[w_{n}(0), w_{n}(1), \ldots, w_{n}(p)\right]^{T}
$$

The error signal or fee characteristic is the difference among the favoured and the anticipated signal 


$$
e(n)=d(n)-\hat{d}(n)
$$

The variable filter out estimates the favoured sign by using convolving the input sign with the impulse reaction. In vector notation that is expressed as $\tilde{d}(n)=\mathbf{w}_{n} * \mathbf{x}(n)$

Where

$\mathbf{x}(n)=[x(n), x(n-1), \ldots, x(n-p)]^{1}$

Is an enter sign vector moreover, the variable filter out updates the filter out coefficients at on every occasion immediately.

$$
\mathbf{w}_{n+1}=\mathbf{w}_{n}+\Delta \mathbf{w}_{n}
$$

Where $\Delta \mathbf{w}_{n}$ is a correction thing for the clear out of the coefficients. The adaptive set of rules generates this correction issue based totally at the input and error signals. LMS and RLS define different coefficient update algorithms.

\section{THE PROPOSED METHOD}

Our approach is based totally on the fundamentals of trimmed imply filter. Inside the case of alpha trimmed mean clear out (ATMF), trimming is symmetric at each ends for the values selected from a $3 \times 3$ window. So there can be a case that together with noisy pixels a few unconverted pixels also trimmed which results inside the lack of picture details. In our case, we first test the processing pixel whether or not its miles corrupted or uncorrupted i.e. the first step is the impulse detection. Whilst the processing pixel is uncorrupted i.e. its gray degree lies between the maximum and minimum grey stage fee then it's far left unchanged. When the processing pixel is corrupted then its miles passed via the proposed filter out. The general filtering technique can be described inside the following steps.

Step-I: A 2-D $3 \times 3$ matrix is selected all over the image pixel that centre of matrix corresponds to processing pixel. Let $\mathrm{f}(\mathrm{ij})$ be pixel under processing.

Step-II: Processing pixel is checked and there arrives two cases.

Case-a: If $0<\mathrm{f}(\mathrm{ij})<255$, then processing pixel left unchanged.
For example if the processing pixel is having a value of 85 shown below, then it is left unchanged.

$\left[\begin{array}{ccc}255 & 0 & 255 \\ 60 & (85) & 78 \\ 0 & 255 & 96\end{array}\right]$

Case-b: If $f(i j)=0$ or $f(i j)=255$ i.e. while the processing pixel is corrupted again conditions arise. condition-l: when all the acquaintances are noisy i.e. they all have either a value of $\mathrm{O}$ or 255 or each then we find the suggest fee of the window and replace the processing pixel with this mean fee. We cross for the mean cost because if we calculate the median however it is going to be a noise. Let us discuss the subsequent state of affairs. Here the processing pixel is 255 method noisy and all of the pals are corrupted i.e. all of them have both a price zero or 255 or both. So we find the mean of the window that's 85 and update the processing pixel with this price.

$$
\left[\begin{array}{ccc}
154 & 255 & 80 \\
255 & (0) & 65 \\
0 & 90 & 146
\end{array}\right]
$$

Here the centre pixel is corrupted (i.e. 0) and a number of the associates have the price of 0 or 255 . So we first dispose of those corrupted values from the window. The 1-zero array illustration of the above matrix will be [154 25580255065090 146]. After the elimination of the noisy pixels it will likely be [154 806590 146]. The mean of these final values may be 107 . The processing pixel will be replaced by using this value. Step-three: Repeat the step-1 and step- 2 until all of the pixels of picture are blanketed.

\section{EXPERIMENTAL RESULTS}

So as to check the performance and effectiveness of the proposed filter we've selected gray scale photo of Lena and cameraman of length 512 X 512 and 256 X 256 respectively. Then we have calculated PSNR and IEF varying the noise density from $10 \%$ to $90 \%$ with boom in density of noise of $10 \%$. Right here the window size is $3 \times 3$ for all the filters that we have in comparison. 
International Journal of Trend in Scientific Research and Development (IJTSRD) ISSN: 2456-6470

TABLEN

PSNR comparison of algorithms with proposed filter for Lena image with different noise densities

\begin{tabular}{|c|l|l|l|l|l|}
\hline \multirow{2}{*}{$\begin{array}{c}\text { Noise } \\
\text { density in } \\
\%\end{array}$} & \multicolumn{5}{|c|}{ PSNR in dB } \\
\cline { 2 - 6 } & MF & AMF & DBA & ATMF & $\begin{array}{c}\text { PROPOSED } \\
\text { FILTER }\end{array}$ \\
\hline 10 & 33.74 & 39.73 & 41.43 & 39.80 & 43.83 \\
\hline 20 & 28.88 & 32.60 & 37.03 & 32.25 & 40.11 \\
\hline 30 & 23.78 & 25.92 & 34.01 & 26.07 & 37.70 \\
\hline 40 & 19.08 & 20.90 & 31.80 & 21.30 & 35.55 \\
\hline 50 & 15.24 & 16.84 & 29.90 & 17.69 & 33.03 \\
\hline 60 & 12.39 & 13.61 & 27.80 & 14.81 & 29.35 \\
\hline 70 & 10.06 & 10.96 & 25.49 & 12.39 & 24.99 \\
\hline 80 & 8.14 & 8.78 & 23.19 & 10.39 & 20.36 \\
\hline 90 & 6.64 & 6.99 & 19.51 & 8.85 & 16.07 \\
\hline
\end{tabular}

TABLE II

IEF comparison of algorithms with proposed filter for Lena image with different noise densities

\begin{tabular}{|c|c|c|c|c|c|}
\hline \multirow{2}{*}{$\begin{array}{c}\text { Noise } \\
\text { density in } \\
\%\end{array}$} & \multicolumn{5}{|c|}{ IEF } \\
\cline { 2 - 6 } & MF & AMF & DBA & ATMF & $\begin{array}{c}\text { PROPOSED } \\
\text { FILTER }\end{array}$ \\
\hline 10 & 67.61 & 266.45 & 396.40 & 273.12 & 690.20 \\
\hline 20 & 44.43 & 103.34 & 289.47 & 95.22 & 585.51 \\
\hline
\end{tabular}

\begin{tabular}{|l|l|l|l|l|l|}
\hline 30 & 20.44 & 33.60 & 216.71 & 34.68 & 493.70 \\
\hline 40 & 9.25 & 14.06 & 173.63 & 15.41 & 406.29 \\
\hline 50 & 4.78 & 6.85 & 139.87 & 8.37 & 286.37 \\
\hline 60 & 2.97 & 3.93 & 103.36 & 5.16 & 147.55 \\
\hline 70 & 2.02 & 2.48 & 70.91 & 3.46 & 62.81 \\
\hline 80 & 1.49 & 1.72 & 47.87 & 2.5 & 24.80 \\
\hline 90 & 1.18 & 1.28 & 22.96 & 1.96 & 10.35 \\
\hline \hline
\end{tabular}

Table 1: PSNR And IEF comparisons of algorithms with proposed filter for Lena Image with different noise densities
TABLE III

PSNR comparison of algorithms with proposed filter for cameraman image with different noise densities

\begin{tabular}{|c|c|c|c|c|c|}
\hline \multirow{2}{*}{$\begin{array}{c}\text { Noise } \\
\text { density in } \\
\%\end{array}$} & \multicolumn{5}{|c|}{ IFF } \\
\cline { 2 - 6 } & MF & AMF & DBA & ATMF & $\begin{array}{c}\text { PROPOSED } \\
\text { FILTER }\end{array}$ \\
\hline 10 & 26.19 & 33.96 & 34.43 & 33.82 & 35.69 \\
\hline 20 & 24.06 & 28.71 & 30.15 & 28.34 & 32.30 \\
\hline 30 & 20.78 & 23.73 & 27.37 & 23.95 & 30.02 \\
\hline 40 & 17.43 & 19.45 & 25.45 & 20.08 & 28.27 \\
\hline 50 & 14.43 & 15.91 & 23.81 & 16.80 & 26.34 \\
\hline 60 & 11.94 & 12.99 & 22.20 & 14.08 & 24.45 \\
\hline 70 & 9.51 & 10.44 & 20.67 & 11.67 & 21.52 \\
\hline 80 & 7.73 & 8.37 & 18.72 & 9.90 & 17.92 \\
\hline 90 & 6.29 & 6.60 & 16.13 & 8.28 & 14.22 \\
\hline
\end{tabular}

TABLE IV

IEF comparison of a gorithms with proposed filter for cameraman image with different noise densities

\begin{tabular}{|c|c|c|c|c|c|}
\hline \multirow{2}{*}{$\begin{array}{c}\text { Noise } \\
\text { density in } \\
\%\end{array}$} & \multicolumn{5}{|c|}{ IEF } \\
\cline { 2 - 6 } & MF & AMF & DBA & ATMF & $\begin{array}{c}\text { PROPOSED } \\
\text { FILTER }\end{array}$ \\
\hline 10 & 12.86 & 75.01 & 85.34 & 74.33 & 114.21 \\
\hline 20 & 15.83 & 45.79 & 63.57 & 41.97 & 105.71 \\
\hline 30 & 11.16 & 21.98 & 51.21 & 23.08 & 93.31 \\
\hline 40 & 6.88 & 11.05 & 43.42 & 12.65 & 83.92 \\
\hline 50 & 4.29 & 6.01 & 37.20 & 7.44 & 66.67 \\
\hline 60 & 2.88 & 3.71 & 28.52 & 4.76 & 52.41 \\
\hline 70 & 1.94 & 2.48 & 25.43 & 3.20 & 31.00 \\
\hline 80 & 1.47 & 1.70 & 18.53 & 2.42 & 15.32 \\
\hline 90 & 1.19 & 1.27 & 11.52 & 1.88 & 7.37 \\
\hline
\end{tabular}

Table 2: PSNR And IEF Comparisons of algorithms with proposed filter for Cameraman Image with different noise densities 
The PSNR and IEF are described by following expressions.

$\mathrm{PSNR}_{\text {in dB }}=10 \log _{10}\left(\frac{255^{2}}{\mathrm{MSE}}\right)$

Where MSE is defined as

$$
\begin{aligned}
& M S E=\frac{\sum_{i=1}^{M} \sum_{j=1}^{N}(I(i, j)-C(i, j))^{2}}{M \times N} \\
& I E F=\frac{\sum_{i=1}^{M} \sum_{j=1}^{N}(A(i, j)-I(i, j))^{2}}{\sum_{i=1}^{M} \sum_{j=1}^{N}(C(i, j)-I(i, j))^{2}}
\end{aligned}
$$

Here MSE stands for Mean Square Error. I(i,j) represents the original image, $\mathrm{C}(\mathrm{i}, \mathrm{j})$ represents the denoised image, and $\mathrm{A}(\mathrm{i}, \mathrm{j})$ denotes the noisy image.
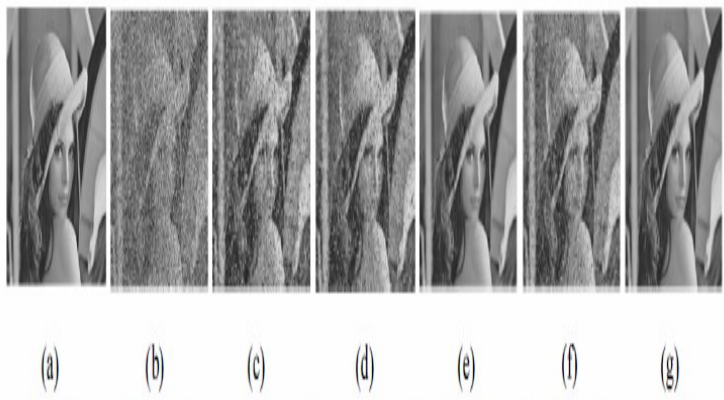

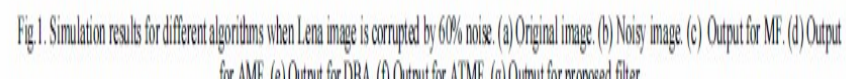

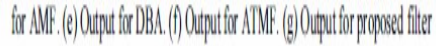
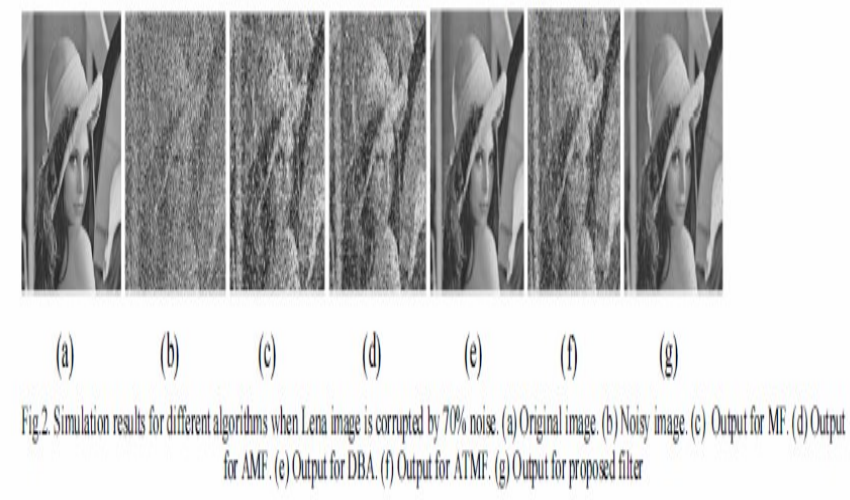

Figure 9: Simulation results for different algorithms when Lena Image is Corrupted by $60 \%$ and $70 \%$
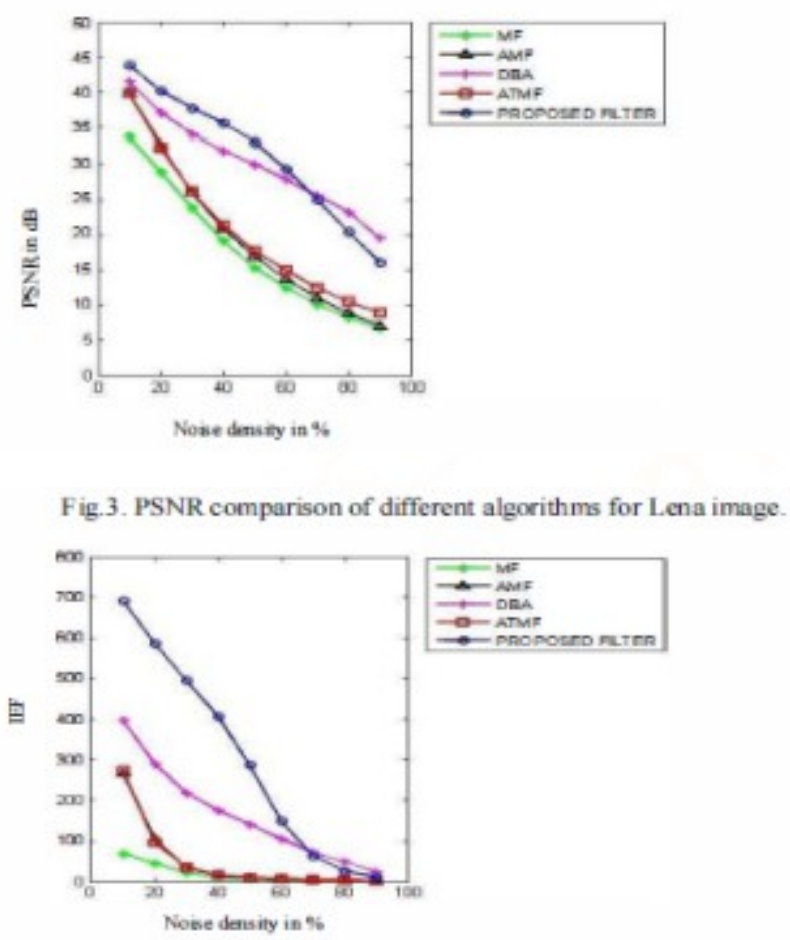

Fig. 4. IEF comparison of different algorithms for Lena image.

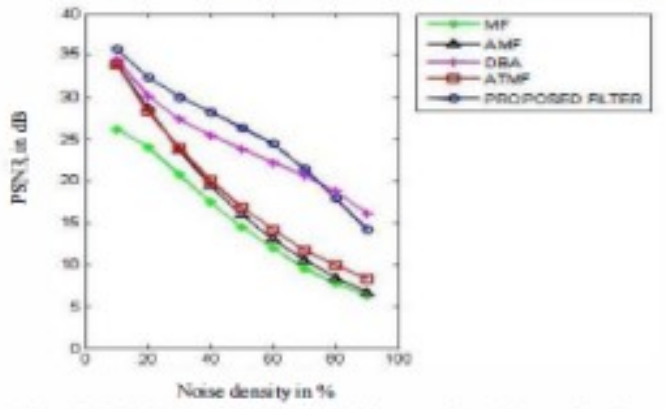

Fig. 5. PSNR comparison of different al gorithms for Cameraman image.

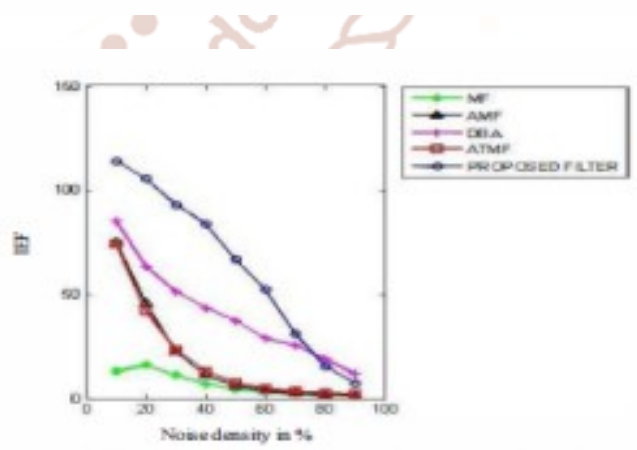

Fig.6. IEF comparison of different al gorithms for Cameraman image

Figures 10: PSNR And IEF Comparisons of different algorithms for Lena Image And Cameraman Image

The noisy snap shots are then surpassed through special median filters in conjunction with the proposed filter out and we get the above output photographs. 
A photo is a -dimensional photograph, which has a similar look to a few concern normally a bodily object or a person. Photo is a two-dimensional, together with a picture, displays, and as well as a three-dimensional, together with a statue. They will be captured via optical devices-consisting of cameras, mirrors, lenses, telescopes, microscopes, and so on. Herbal items and phenomena, along with the human eye or water surfaces. The word image is also used within the broader experience of any -dimensional parent together with a map, a graph, a pie chart, or an abstract painting. in this wider experience, photographs can also be rendered manually, including by way of drawing, portray, carving, rendered routinely with the aid of printing or laptop images generation, or advanced through a combination of techniques, mainly in a pseudo-photo.

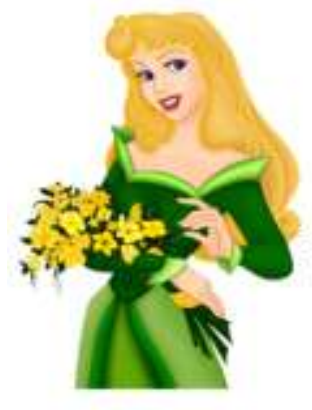

RGB image

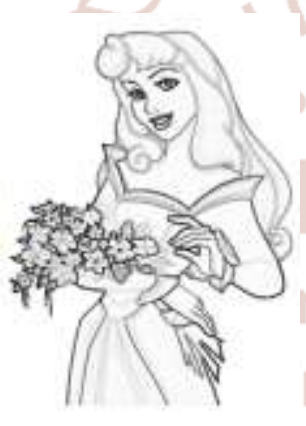

Gary colour image
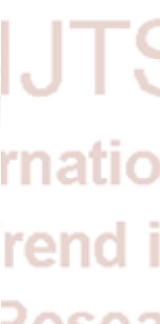

\section{Figures 11: RGB Image And Gray Colour Image}

A photograph is a square grid of pixels. It has a exact peak and a precise width counted in pixels. Each pixel is square and has a hard and fast size on a given display. But one-of-a-kind laptop monitors may also use unique sized pixels.

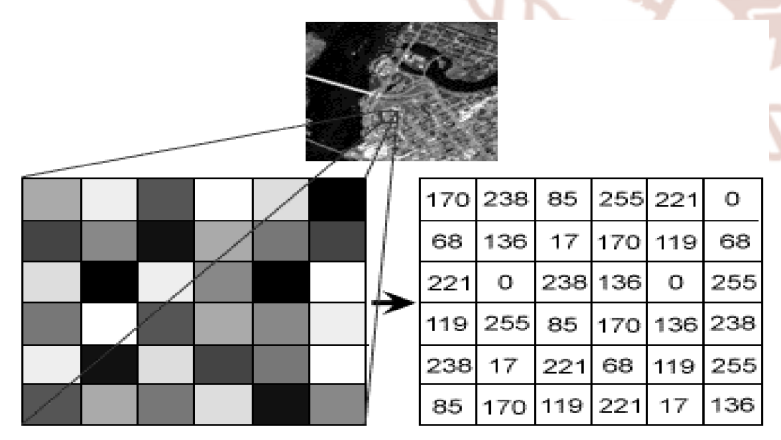

Figure 12: Image Converted to Pixels

Each pixel has a shade. The coloration is a 32-bit integer. The first eight bits decide the redness of the pixel, the subsequent 8 bits the greenness, the following eight bits the blueness, and the ultimate 8 bits the transparency of the pixel.

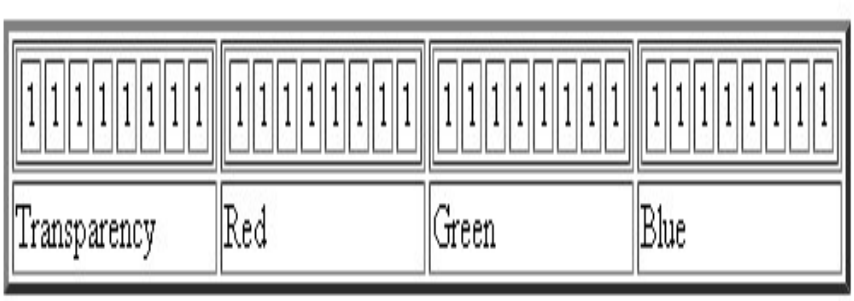

\section{Table: 3: 8Bits of an RGB Transparency Image}

Picture file size is expressed as the number of bytes that increases with the range of pixels composing a picture, and the shade intensity of the pixels. The more the number of rows and columns, the extra the picture resolution, and the bigger the document. Also, each pixel of a photograph will increase in length whilst its colour intensity will increase, an 8-bit pixel ( 1 byte) stores 256 hues, a 24-bit pixel (3 bytes) stores 16 million colours, the latter known as true colour. Photograph compression uses algorithms to decrease the size of a document. Excessive resolution cameras produce large picture files, starting from hundreds of kilobytes to megabytes, per the digital camera's decision and the photograph-storage layout potential. High resolution virtual cameras record 12 megapixel ( $1 \mathrm{MP}=1,000,000$ pixels / 1 million $)$ photographs, or more, in proper coloration. as an example, an image recorded via a 12 MP digicam; considering that each pixel uses 3 bytes to record authentic colour, the uncompressed photograph could occupy 36,000,000 bytes of reminiscence, a top notch amount of virtual storage for one photo, given that cameras have to file and shop many images to be realistic. confronted with huge record sizes, both in the digicam and a garage disc, picture record codecs had been advanced to store such big photographs.

\section{CONCLUSION \& FUTURE SCOPE}

In our proposed system we successfully implemented the new filter method to improve the rectification of the result of the images which are affected by the salt and pepper noise . This filter indicates better end result than suggest filter out like ATMF and versions of MF in phrases of PSNR and IEF. The proposed clear out is tested for exceptional densities of noise and it shows consistent end result over the range of noise densities. In older we are losing the edge data and our system shown the improved results when compared to visual results and also PSNR, MSE. The proposed set of rules is quicker because it uses a small window of size $(3,3)$. 
In addition, it influences a clean transition among the pixel values by way of utilizing the correlation between neighbouring processed pixels at the same time as keeping facet details as a consequence leading to higher part protection. The performance of the set of rules has been tested at low, medium and high noise densities on both grey-scale in addition to shade pix. Even at high noise density degrees the PA gives higher outcomes in assessment with different existing algorithms. Computation time is reduced significantly as compared with stage algorithm of Chan etc.

\section{REFERENCES}

1. L. S. Srinivasan, D. Ebenezer, "A New Fast and Efficient Decision-Based Algorithm for Removal of High-Density Impulse Noise" IEEE Signal Processing Paperes, Vol. 14, No. 3, pp. 189-192, March 2007

2. R. H. Chan, Chung-WaHo, M. Nikolova, "Salt and Pepper Noise Removal by Median Type Noise Detectors and Detail -Preserving Regularization," IEEE Transactions on Image Processing, Vol. 14,No.10, pp. 1479-1485, October 2005.

3. T. S. Huang, G. J. Yang, and G. Y. Tang, "Fast two-dimensional median filtering algorithm," IEEE Trans. Acoustics, Speech, Signal Process., Vol. ASSP-1, No. 1, pp. 13-18, Jan. 1979.

4. C. A. Pomalaza_Racz and C. D. Macgillem, "An adaptive nonlinear edge preserving filter," IEEE Trans. Acoustics, Speech, Signal Process., Vol. ASSP-32, pp.571-576, Jun. 1984.

5. T. Sun and Y. Neuvo, "Detail-preserving median based filters in image processing," Pattern Recognition. Lett., vol. 15, pp. 341-347, 1994.

6. H.-L. Eng and K.-K. Ma, "noise adaptive softswitching median filter,", IEEE Trans. Image Process., vol. 10, no. 2, pp. 242-251, Feb. 2001.

7. Haidi Ibrahim, NieholasSiaPik Kong, and Theam Foo Ng, "Simple Adaptive Median Filter for the Removal of Impulse Noise from HighlyCorrupted Images," IEEE Trans Consumer Electronics, Vol. 54, No. 4,pp. 1920-1927, November 2008.

8. S. Zhang and M. A. Karim, "A new impulse detector for switching median filters," IEEE Signal Process. Lelt., vol. 9, no. 11, pp. 360363,Nov. 2002.
9. Z Wang and D. Zhang, "Progressive switching median filter tor the removal of impulse noise from highly corrupted images," IEEE Trans. Circuits Syst. II., Analog Digit. Signal Process, vol. 46, no. 1, pp. 78-80,Jan. 1999.

10. V. Jayaraj and D. Ebenezer, "A new switchingbased median filtering scheme and algorithm tor removal of high-density salt and pepper noisein image," EURASIP J. Adv. Signal Process, 2010.

11. S. Esakkirajan, T. Veerakumar, Adabala N. Subramanyam, and C. H. PremChand, "Rem oval of High Density Salt and Pepper Noise Through Modified Decision Based Unsymmetric Trimmed Median Filter," IEEE Signal Process Lelt., vol. 18, no. 5, pp. 287-290, May 2011.

12. Yadav, P Electron. \&Commun.,Laxmipatiinstn. Of Sci. \& Technol., Bhopal, india "Colour image noise removal by modified adaptive threshold median filter for RVIN"

13. Lim, Jae S., Two-Dimensional Signal and Image Processing, Englewood Cliffs, NJ, Prentice Hall, 1990, pp. 469-476

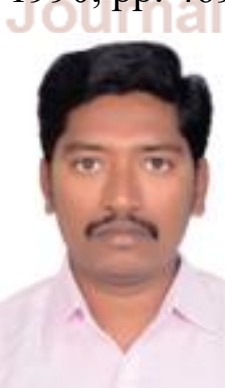

Sathuluri Ramarao, M.Tech Scolar, Digital Image Processing, Nalanda Institute of Engineering and Technology (NIET), Guntur District, Andhra Pradesh, India, ramarao409@gmail.com

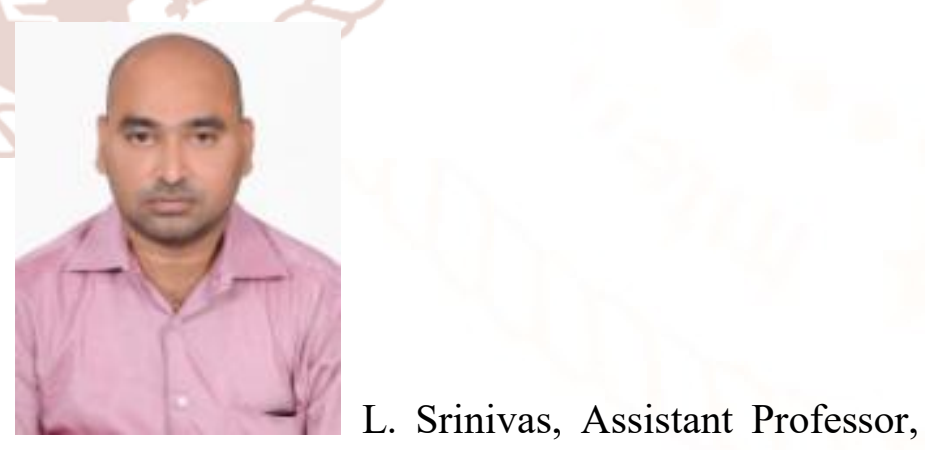
Digital Image Processing, Nalanda Institute of Engineering and Technology (NIET), Guntur District, Andhra Pradesh, India. 